\title{
De-escalation of Systemic Therapy for Early-Stage, Node-Negative Her2+ and Triple-Negative Breast Cancer
}

\author{
Lorena Gonzalez $^{1}$ (D) Joanne Mortimer ${ }^{2} \cdot$ Laura Kruper $^{1}$
}

Accepted: 19 May 2021 / Published online: 14 June 2021

(C) The Author(s) 2021

\begin{abstract}
Purpose of Review This review summarizes the most recent data on the management of small, node-negative Her2+ and triplenegative breast cancer.

Recent Findings Both Her2+ and triple-negative breast cancers are characterized by high rates of recurrence and worse survival outcomes compared to hormone-positive cancers. De-escalation of systemic therapy in early-stage breast cancer is a recent national trend in clinical research. Recent prospective trials support the scaling back of cytotoxic agents and maximization of targeted therapy regimens. Similarly, large retrospective studies on small, node-negative triple-negative breast cancer report the omission of chemotherapy in women with T1a,N0 triple-negative cancers with favorable short term outcomes.

Summary De-escalation of systemic therapy for Her2+ breast cancer is effective in the management of early-stage, node-negative disease. Future prospective studies on the omission of systemic therapy for triple-negative breast cancer are required to safely adopt into consensus guidelines.
\end{abstract}

Keywords Systemic chemotherapy $\cdot$ Her2+ breast cancer $\cdot$ Triple-negative breast cancer $\cdot$ T1a,bN0

\section{Introduction}

The incidence of stage I breast cancers has increased significantly, comprising almost $50 \%$ of all newly diagnosed nonmetastatic breast cancers $[1,2]$. This finding is largely due to screening mammography, which has appreciably increased the rate of detection of non-palpable breast cancers, especially $\mathrm{T} 1$ tumors $\leq 1 \mathrm{~cm}[1,2]$. Though outcomes for women with T1a,bN0M0 disease is favorable (breast cancer-specific survival $\geq 95 \%$ at 10 years [3-5]), treatment decision making for these patients remains challenging. The advent of the 21 -gene RT-PCR assay has provided significant insight into the management of $\mathrm{T} 1$ estrogen receptor (ER) positive cancers with

This article is part of the Topical Collection on Local-Regional Evaluation and Therapy

Lorena Gonzalez

loregonzalez@coh.org

1 Department of Surgery, Division of Breast Surgery, City of Hope National Medical Center, 1500 E Duarte Rd, Duarte, CA 91010, USA

2 Department of Medical Oncology, City of Hope National Medical Center, Duarte, CA, USA respect to systemic treatment $[6,7]$. As a whole, sub-centimeter, lymph node-negative breast cancers have an excellent prognosis, but the absence of estrogen and progesterone hormone receptors (HR) is associated with a higher breast cancerspecific mortality suggesting that outcomes for even the smallest of tumors are still very much dependent on tumor biology $[5,8]$. Patients with human epidermal growth factor receptor 2 positive (HER2+) or triple-negative (TN; estrogen receptor, progesterone receptor and HER2-negative) breast cancers have worse disease-free survival overall, but those with node-negative T1a,b tumors have DFS $>90 \%$, even without adjuvant systemic therapy [8]. Despite the robust development of novel targeted therapies bolstering the arsenal of systemic treatment for Her2+ disease, patients with these small tumors have been traditionally poorly represented or excluded in the pivotal adjuvant chemotherapy trials [9-12] resulting in limited evidence for the use of powerful cytotoxic regimens in these patients. Furthermore, pathologic complete response after neoadjuvant chemotherapy has become not only a prognostic factor for patients with TNBC but also helps identify patients who benefit from specific adjuvant therapies in cases of residual disease [13]. Here, we review the latest evidence for the current treatment of both sub-centimeter, node-negative Her2+ and triple-negative breast cancers and 
discuss novel approaches for their management in the context of the existing international consensus treatment guidelines.

\section{Early-Stage HER2+ Breast Cancers}

\section{Single Agent Chemotherapy}

While the addition of trastuzumab to adjuvant chemotherapy has been a landmark achievement in the treatment of Her2+ disease, data suggest that select patients may have equal outcomes with less intense therapy. Clinical factors such as tumor size and nodal status may be adequate for identifying such patients; however, a large retrospective study of $\leq 1 \mathrm{~cm}$ Her2+ breast cancers show that even small tumors that are lymph node negative have a high risk of recurrence spanning up to $23 \%$ at 5 years when adjuvant trastuzumab-based chemotherapy is omitted [14]. As previously mentioned, patients with tumors $<2 \mathrm{~cm}$ were generally excluded from the largescale pivotal trials of targeted HER2+ therapies [9-12]. Since then, two large prospective trials of alternative less aggressive adjuvant chemotherapy regimens were performed in patients with small tumors.

In 2015, the 4-year follow-up results of the Adjuvant Paclitaxel Trastuzumab (APT) trial were released showing a 98.7\% disease free survival (DFS) in patients with $\leq 3 \mathrm{~cm}$ lesions. This trial was an uncontrolled, single-armed, multicenter trial of adjuvant paclitaxel and trastuzumab in 406 patients with node-negative Her $2+$ tumors measuring $\leq 3 \mathrm{~cm}$ [15]. The intervention in this prospective study consisted of adjuvant weekly paclitaxel $\left(80 \mathrm{mg} / \mathrm{m}^{2}\right)$ and trastuzumab for 12 weeks followed by weekly trastuzumab for another 40 weeks to complete a year of treatment. The updated 7 year follow-up data released in 2019 revised that DFS to 93.3\%; however, this change in DFS is still a favorable outcome for a malignancy that used to carry a recurrence risk of $>20 \%$ in the pre-trastuzumab era. The second trial performed in 2013 was a phase 2 single-armed trial of a second non-anthracycline based chemotherapy regimen in association with targeted therapy for HER2+ cancers in an adjuvant fashion [16]. This prospective study of 493 patients with early-stage Her2+ disease showed a similar 98\% 3-year DFS for node-negative patients after treatment with docetaxel and cyclophosphamide plus trastuzumab. Both trials laid the groundwork for even further de-escalation of treatment for early-stage HER2+ cancers, including the possibility of omitting cytotoxic chemotherapy from treatment regimens completely.

\section{Chemotherapy-Free Regimens}

Optimal targeted therapy with dual anti-Her2 therapy without chemotherapy has been shown to be effective in a select group of Her2+ tumors with or without endocrine therapy [17]. A single armed trial of 66 patients with stage II-III Her2+ tumors were treated with weekly trastuzumab and daily lapatinib for 12 weeks. Patients with ER-positive tumors in this study also received neoadjuvant endocrine therapy with letrozole and ovarian suppression if premenopausal. The pathologic complete response (pCR) in ER positive tumors was $21 \%$ and $36 \%$ in ER-negative disease. These findings suggest that there is a specific group of early-stage Her2+ tumors which are being over treated and could benefit from further de-escalation of treatment by eliminating chemotherapy completely. Five years later, the NAPHER-2 trial evaluated another chemotherapy free regimen of dual anti-HER 2 therapy with trastuzumab and pertuzumab plus palbociclib and fulvestrant in 35 patients with HER 2+, ER+ breast cancers. At surgery, patients achieved a breast and lymph node pCR of $27 \%$. This finding is comparable to the pCR achieved in the NeoSphere trial with dual anti-HER2 therapy and paclitaxel [18] again supporting the argument for maximum targeted-therapy (chemotherapyfree) regimens.

Recently, the results of the ATEMPT trial, a randomized, trial comparing outcomes after targeted therapy with traditional cytotoxic therapy regimens for Her2+ tumors, were released. This trial compared adjuvant trastuzumab emtansine TDM-1 to paclitaxel plus trastuzumab for the treatment of early HER2+ cancers and was presented in December of 2019 at the San Antonio Breast Cancer Symposium [19]. Use of single agent adjuvant TDM-1 resulted in a 3-year disease-free survival (DFS) of $97.7 \%$ and 3-year recurrence-free interval of $99.1 \%$. Of note, in this study DFS did not differ based on hormone receptor status as they did in a previous study of targeted therapy alone [17]. The overall DFS of 97.7\% included $97.5 \%$ of patients with HR-positive disease and $98.5 \%$ of patients with HR-negative disease. Tumor size also did not affect DFS $(98.5 \%$ for tumors $<1 \mathrm{~cm} ; 97.1 \%$ for tumors $\geq 1 \mathrm{~cm}$ ). The paclitaxel plus trastuzumab arm had a 3year DFS of $92.8 \%$, significantly lower than that of TDM-1 alone. Furthermore, the 2 treatment arms in the ATEMPT trial did not differ with respect to clinically relevant toxicities, the other primary endpoint of this phase 2 trial. As further evidence for neoadjuvant targeted therapy alone in early-stage HER2+ tumors, future research should focus on the identification of predictive biomarkers of response to these therapies to better select patients who would benefit from these less intense treatment regimens.

\section{Adjuvant Therapy for Residual Small HER2+ Tumors}

Practice changing studies have also recently been reported for patients with residual HER2+ tumors that are small in size. The KATHERINE trial [20] was a phase III study of HER2+ early-stage breast cancers treated with neoadjuvant 
chemotherapy and trastuzumab, followed by surgery. Patients with residual disease (including patients with < $1 \mathrm{~cm}$ of residual disease) were then randomized either to TDM1 or more trastuzumab for another 14 cycles. Their primary endpoint was invasive DFS. Overall, there was an $11 \%$ absolute improvement in invasive DFS survival between the treatment groups. Relevant to this review of small HER2+ tumors, a pre-specified subgroup analysis performed showed that patients with node-negative disease ( $\mathrm{HR}=0.44 ; 95 \% \mathrm{CI}$ $0.28-0.68)$, as well as those with very small residual disease $(<1 \mathrm{~cm})(\mathrm{HR}=0.6 ; 95 \%$ CI $0.33-1.12)$ benefitted from adjuvant TDM-1 compared to standard therapy. TDM-1 therapy for residual HER2+ disease after neoadjuvant therapy, irrespective of size, is now standard therapy for patients who can tolerate its side effect profile (significantly more thrombocytopenia, peripheral neuropathy, and transaminasemia).

\section{Early-Stage Triple-Negative Breast Cancers (TNBC)}

Retrospective reviews of all patients with T1a,bN0M0 stage breast cancer show a 10 -year recurrence free survival ranging from 82 to $98 \%$. Studies of breast cancer screening [4, 21, 22] and the SEER database $[23,24]$ report comparable survival rates for these small cancers but were worse in patients with specific high-risk features such as age greater than 50 with ER-negative tumors or those with high-grade tumors. Tenyear overall survival is $24 \%$ and cause-specific mortality is $4 \%$ for patients with early-stage breast carcinoma in the SEER database for patients from 1988 to 2001 [3]. Again, the characteristics associated with worse outcomes were young age $(\leq 50)$, high tumor grade, ER-negative status, and PR-negative status.

Treatment for hormone-receptor negative small tumors has been ambiguous. In patients with $\mathrm{T} 1 \mathrm{a} / \mathrm{bN} 0$ tumors treated with $[25,26]$ and without $[27,28]$ adjuvant chemotherapy, ER-negative tumors had worse outcomes independent of treatment in several studies. One study also found that the reduced 5-year recurrence free survival in ER-negative small, node-negative tumors was no longer present at 10year follow-up [28]. However, outcome data for patients with early-stage invasive breast cancer enrolled in 5 National Surgical Adjuvant Breast and Bowel (NSABP) randomized trials found that the recurrence-free survival rate was increased by almost $10 \%$ in patients with ER-negative cancers receiving adjuvant chemotherapy compared to those receiving surgery alone [29].

Two contemporary cohort studies from Korea and Italy $[30,31]$ of early-stage node-negative breast cancers found that early-stage TNBC patients had higher risk of recurrence, especially in the very young (age less than 35 years). In the
Italian study, over $66 \%$ of TNBC patients had some form of adjuvant chemotherapy and $>90 \%$ received some form of radiotherapy. They noted that TNBC patients were 3.5 times more likely to develop loco-regional recurrence and women with Her2+ tumors were 4.5 times more likely to recur. Similarly, in a large US based cohort study of exclusively $\mathrm{T} 1 \mathrm{a} / \mathrm{bN} 0 \mathrm{M} 0 \mathrm{TNBC}$ patients at a large single institution, $58 \%$ of women received adjuvant chemotherapy and $63 \%$ received whole-breast radiation [32]. Those who received chemotherapy in this study were younger, had larger tumors, or had higher histologic grade; therefore, treatment assignment was not random. Neither locoregional recurrence-free survival nor distant metastasis-free survival was affected by tumor size (T1mic/T1a vs. T1b). Receipt of adjuvant chemotherapy also had no effect and both locoregional recurrence and distant metastasis were low $(<5 \%)$. It is difficult to discern from this study whether the favorable prognosis observed in the small was due to the effect of the chemotherapy or an inherent low metastatic potential of small, nodenegative TNBC. A retrospective study of outcomes for small, node-negative invasive breast cancer that included over 350 T1a,bN0 TNBC patients from the NCCN database showed similar findings [8]. This study found 5-year distant relapse-free survivals of $93 \%$ (without adjuvant chemotherapy) to $100 \%$ (with adjuvant chemotherapy) for T1a tumors and $90 \%$ (without adjuvant chemotherapy) to $96 \%$ (with adjuvant chemotherapy) for T1b tumors. This data supports the finding that as with Her2+ tumors, there is a group of women with small, node-negative TNBC that is being over treated with adjuvant systemic therapy. The challenge lies in identifying and selecting good responders in order to avoid toxic effects to the non-responders. Conversely, a more recent retrospective review of the National Cancer Data Base ( $\mathrm{n}=$ 13,065) investigating the use of adjuvant chemotherapy in stage IA TNBC patients reports most of these patients are receiving adjuvant chemotherapy, including $48 \%$ of patients with T1a tumors [33]. The authors further demonstrated better 4-year OS benefit in patients with all T1 tumors, but this improvement was not statistically significant for $\mathrm{T} 1 \mathrm{a}$ lesions.

In the absence of prospective trials on the role of adjuvant systemic therapy for small, node-negative TNBC, the available retrospective data suggest that the use of patient (age) and tumor-related characteristics are useful in guiding the use of chemotherapy in this population. However, the decision to use chemotherapy in this subset of patients still varies between institutions and is likely due to the fact that the current national and international guidelines differ in their recommendations for adjuvant systemic therapy for small, node-negative TNBC. The most recent consensus guidelines of the NCCN recommend no adjuvant therapy for T1a, nodenegative TNBC and to consider systemic therapy for T1a TNBCs with micrometastases as well as for T1b tumors [34]. These guidelines are routinely updated and specifically 
address the management of both $\mathrm{T} 1 \mathrm{a}$ and $\mathrm{T} 1 \mathrm{~b}$ node-negative cancers that are triple negative separately. The NCCNpreferred chemotherapy regimens may or may not be anthracycline based. The majority of panelists of the St. Gallen International Consensus Guidelines for primary therapy of early breast cancer prefer taxane- and alkylator-based systemic therapy rather than anthracyclines for stage T1a,bN0 TNBC and recommend chemotherapy be given to patients with tumors $<0.5 \mathrm{~cm}$ on a "case-by-case" basis [35]. Similarly, the National Institutes of Health recommend individualizing the decision to administer systemic therapy to women with lymph node-negative cancers that are smaller than $1 \mathrm{~cm}$ but do not specify recommendations based on receptor status [36].

This discrepancy in consensus guidelines complicates treatment decisions for patients with T1a,bN0M0 TNBC breast cancer. Recently, a group from the Netherlands presented findings from the largest cohort to date ( $>4000)$ of earlystage, node-negative TNBCs from the Netherlands Cancer Registry [37]. They compared overall survival (OS) and breast cancer-specific survival (BCSS) in patients undergoing systemic therapy to those who did not. More than half (53\%) of the cohort received chemotherapy which positively correlated with tumor size. Systemic therapy use was also found to increase over time. Treatment assignment was not random and patients receiving chemotherapy were younger, had larger and higher-grade tumors, and were also found to have more isolated tumor cells in their lymph nodes. At 7 years of follow-up chemotherapy was associated with improved OS and BCSS in the whole group (T1a-cN0). After adjuvant chemotherapy, only patients with $\mathrm{T} 1 \mathrm{c}$ and grade 3 tumors had significantly improved OS and BCSS rates. The authors estimated an absolute difference of 14-16\% for both OS and BCSS in patients with high-grade $\mathrm{pT} 1 \mathrm{cNO}(\mathrm{itc}+) \mathrm{TNBC}$ with the use of adjuvant systemic therapy while they observed no difference in OS nor BCSS in those with smaller TNBCs or those with grade 1-2 tumors. This large, contemporary cohort study supports the omission of adjuvant systemic therapy for sub-centimeter, node-negative TNBC and differs from the current NCCN guidelines.

Finally, it is important to note that the guidelines do not equivocate on the management of residual TNBC after neoadjuvant chemotherapy. Patients with TNBC who do not attain a pathologic complete response have a $20-30 \%$ rate of recurrence. To this effect, the CREATE-X trial randomized Her2-negative patients with residual disease after neoadjuvant chemotherapy to receive standard therapy + capecitabine vs. standard therapy alone. Among patients with TNBC the rate of DFS was $69.8 \%$ compared to $56.1 \%$ in the control group [13]. Overall survival as also significantly improved (78.8\% vs. $70.3 \%$ ). Current NCCN guidelines recommend 6-8 cycles of capecitabine for patients with TNBC who did not achieve a ypT0 response to neoadjuvant therapy.

\section{Conclusion}

In summary, excellent local and distant recurrence control rates are achievable in sub-centimeter, node-negative HER2+ and TNBC with modern systemic targeted therapy and traditional multimodal therapy, respectively. The trend towards de-escalation of treatment has led to the identification of certain subgroups of patients with these early-stage breast cancers that can safely be spared the extreme cytotoxic effects of systemic therapies. Early detection of these tumors remains critical, however, to ensure good short-term outcomes. Furthermore, identification of predictive factors for response to systemic therapies in HER2+ and TNBC will be critical in the development of precision medicine for patients with HR negative early-stage breast cancer. Longer observation of the treatment de-escalation studies will ultimately determine whether these promising short-term outcomes will also result in long-term survival for HER2+ cancers. Future randomized prospective studies for patients with T1a,bN0 TNBC will be necessary to identify who will benefit from systemic therapy in this group.

\section{Declarations}

Conflict of Interest Lorena Gonzalez, Joanne Mortimer, and Laura Kruper declare that they have no conflict of interest.

Human and Animal Rights and Informed Consent This article does not contain any studies with human or animal subjects performed by any of the authors.

Open Access This article is licensed under a Creative Commons Attribution 4.0 International License, which permits use, sharing, adaptation, distribution and reproduction in any medium or format, as long as you give appropriate credit to the original author(s) and the source, provide a link to the Creative Commons licence, and indicate if changes were made. The images or other third party material in this article are included in the article's Creative Commons licence, unless indicated otherwise in a credit line to the material. If material is not included in the article's Creative Commons licence and your intended use is not permitted by statutory regulation or exceeds the permitted use, you will need to obtain permission directly from the copyright holder. To view a copy of this licence, visit http://creativecommons.org/licenses/by/4.0/.

\section{References}

1. Fracheboud J, et al. Nation-wide breast cancer screening in The Netherlands: results of initial and subsequent screening 19901995. National Evaluation Team for Breast Cancer Screening. Int J Cancer. 1998;75(5):694-8.

2. Berry DA, Cronin KA, Plevritis SK, Fryback DG, Clarke L, Zelen $\mathrm{M}$, et al. Effect of screening and adjuvant therapy on mortality from breast cancer. N Engl J Med. 2005;353(17):1784-92.

3. Hanrahan EO, Gonzalez-Angulo AM, Giordano SH, Rouzier R, Broglio KR, Hortobagyi GN, et al. Overall survival and cause- 
specific mortality of patients with stage T1a,bN0M0 breast carcinoma. J Clin Oncol. 2007;25(31):4952-60.

4. Smart CR, Hartmann WH, Beahrs OH, Garfinkel L. Insights into breast cancer screening of younger women. Evidence from the 14year follow-up of the Breast Cancer Detection Demonstration Project. Cancer. 1993;72(4 Suppl):1449-56.

5. Park YH, Kim ST, Cho EY, Choi YL, Ok ON, Baek HJ, et al. A risk stratification by hormonal receptors (ER, PgR) and HER-2 status in small $(<$ or $=1 \mathrm{~cm})$ invasive breast cancer: who might be possible candidates for adjuvant treatment? Breast Cancer Res Treat. 2010;119(3):653-61.

6. Sparano JA, Gray RJ, Makower DF, Pritchard KI, Albain KS, Hayes DF, et al. Adjuvant chemotherapy guided by a 21-gene expression assay in breast cancer. N Engl J Med. 2018;379(2):11121.

7. Paik S, Tang G, Shak S, Kim C, Baker J, Kim W, et al. Gene expression and benefit of chemotherapy in women with node-negative, estrogen receptor-positive breast cancer. J Clin Oncol. 2006;24(23):3726-34.

8. Vaz-Luis I, Ottesen RA, Hughes ME, Mamet R, Burstein HJ, Edge $\mathrm{SB}$, et al. Outcomes by tumor subtype and treatment pattern in women with small, node-negative breast cancer: a multiinstitutional study. J Clin Oncol. 2014;32(20):2142-50.

9. Romond EH, Perez EA, Bryant J, Suman VJ, Geyer CE Jr, Davidson NE, et al. Trastuzumab plus adjuvant chemotherapy for operable HER2-positive breast cancer. N Engl J Med. 2005;353(16):1673-84.

10. Piccart-Gebhart MJ, Procter M, Leyland-Jones B, Goldhirsch A, Untch M, Smith I, et al. Trastuzumab after adjuvant chemotherapy in HER2-positive breast cancer. N Engl J Med. 2005;353(16): 1659-72.

11. Slamon D, Eiermann W, Robert N, Pienkowski T, Martin M, Press M, et al. Adjuvant trastuzumab in HER2-positive breast cancer. N Engl J Med. 2011;365(14):1273-83.

12. Goldhirsch A, Gelber RD, Piccart-Gebhart MJ, de Azambuja E, Procter M, Suter TM, et al. 2 years versus 1 year of adjuvant trastuzumab for HER2-positive breast cancer (HERA): an openlabel, randomised controlled trial. Lancet. 2013;382(9897):1021-8.

13. Masuda N, Lee SJ, Ohtani S, Im YH, Lee ES, Yokota I, et al. Adjuvant capecitabine for breast cancer after preoperative chemotherapy. N Engl J Med. 2017;376(22):2147-59.

14. Gonzalez-Angulo AM, Litton JK, Broglio KR, Meric-Bernstam F, Rakkhit R, Cardoso F, et al. High risk of recurrence for patients with breast cancer who have human epidermal growth factor receptor 2-positive, node-negative tumors $1 \mathrm{~cm}$ or smaller. J Clin Oncol. 2009;27(34):5700-6.

15. Tolaney SM, Barry WT, Dang CT, Yardley DA, Moy B, Marcom PK, et al. Adjuvant paclitaxel and trastuzumab for node-negative, HER2-positive breast cancer. N Engl J Med. 2015;372(2):134-41.

16. Jones SE, Collea R, Paul D, Sedlacek S, Favret AM, Gore I Jr, et al. Adjuvant docetaxel and cyclophosphamide plus trastuzumab in patients with HER2-amplified early stage breast cancer: a singlegroup, open-label, phase 2 study. Lancet Oncol. 2013;14(11): 1121-8.

17. Rimawi MF, Mayer IA, Forero A, Nanda R, Goetz MP, Rodriguez AA, et al. Multicenter phase II study of neoadjuvant lapatinib and trastuzumab with hormonal therapy and without chemotherapy in patients with human epidermal growth factor receptor 2overexpressing breast cancer: TBCRC 006. J Clin Oncol. 2013;31(14):1726-31.

18. Gianni L, Pienkowski T, Im YH, Roman L, Tseng LM, Liu MC, et al. Efficacy and safety of neoadjuvant pertuzumab and trastuzumab in women with locally advanced, inflammatory, or early HER2-positive breast cancer (NeoSphere): a randomised multicentre, open-label, phase 2 trial. Lancet Oncol. 2012;13(1): $25-32$.
19. Tolaney SM, Trippa L, Barry W, Hu J, Dang C, Yardley D, Isakoff S, Valero V, Faggen M, Mulvey T, Bose R, Weckstein D, et al. TBCRC 033: a randomized phase II study of adjuvant trastuzumab emtansine (T-DM1) vs paclitaxel (T) in combination with trastuzumab $(\mathrm{H})$ for stage I HER2positive breast cancer (BC) (ATEMPT). San Antonio Breast Cnacer Symposium. 2020. 80.

20. von Minckwitz G, Huang CS, Mano MS, Loibl S, Mamounas EP, Untch M, et al. Trastuzumab emtansine for residual invasive HER2-positive breast cancer. N Engl J Med. 2019;380(7): 617-28.

21. Seidman H, Gelb SK, Silverberg E, LaVerda N, Lubera JA. Survival experience in the Breast Cancer Detection Demonstration Project. CA Cancer J Clin. 1987;37(5):25890.

22. Smart CR, Byrne C, Smith RA, Garfinkel L, Letton AH, Dodd GD, et al. Twenty-year follow-up of the breast cancers diagnosed during the Breast Cancer Detection Demonstration Project. CA Cancer J Clin. 1997;47(3):134-49.

23. Carter CL, Allen C, Henson DE. Relation of tumor size, lymph node status, and survival in 24,740 breast cancer cases. Cancer. 1989;63(1):181-7.

24. Mirza AN, Mirza NQ, Vlastos G, Singletary SE. Prognostic factors in node-negative breast cancer: a review of studies with sample size more than 200 and follow-up more than 5 years. Ann Surg. 2002;235(1):10-26.

25. Colleoni M, Rotmensz N, Peruzzotti G, Maisonneuve P, Viale G, Renne G, et al. Minimal and small size invasive breast cancer with no axillary lymph node involvement: the need for tailored adjuvant therapies. Ann Oncol. 2004;15(11):1633-9.

26. Lee $\mathrm{AK}$, et al. Lymph node negative invasive breast carcinoma 1 centimeter or less in size (T1a,bNOMO): clinicopathologic features and outcome. Cancer. 1997;79(4):761-71.

27. Crowe JP Jr, et al. Primary tumor size. Relevance to breast cancer survival. Arch Surg. 1992;127(8):910-5 discussion 915-6.

28. Chia SK, Speers CH, Bryce CJ, Hayes MM, Olivotto IA. Tenyear outcomes in a population-based cohort of node-negative, lymphatic, and vascular invasion-negative early breast cancers without adjuvant systemic therapies. J Clin Oncol. 2004;22(9): $1630-7$

29. Fisher B, Dignam J, Tan-Chiu E, Anderson S, Fisher ER, Wittliff $\mathrm{JL}$, et al. Prognosis and treatment of patients with breast tumors of one centimeter or less and negative axillary lymph nodes. J Natl Cancer Inst. 2001;93(2):112-20.

30. Kwon JH, Kim YJ, Lee KW, Oh DY, Park SY, Kim JH, et al. Triple negativity and young age as prognostic factors in lymph nodenegative invasive ductal carcinoma of $1 \mathrm{~cm}$ or less. BMC Cancer. 2010;10:557.

31. Cancello G, Maisonneuve P, Rotmensz N, Viale G, Mastropasqua MG, Pruneri G, et al. Prognosis in women with small (T1mic,T1a, T 1b) node-negative operable breast cancer by immunohistochemically selected subtypes. Breast Cancer Res Treat. 2011;127(3):713-20.

32. Ho AY, Gupta G, King TA, Perez CA, Patil SM, Rogers KH, et al. Favorable prognosis in patients with $\mathrm{T} 1 \mathrm{a} / \mathrm{T} 1 \mathrm{bN} 0$ triple-negative breast cancers treated with multimodality therapy. Cancer. 2012;118(20):4944-52.

33. Patel AN, Peddi SR, Burton GV. Abstract P5-14-02: triple negative breast cancer - adjuvant chemotherapy use and survival outcomes in stage IA disease. Cancer Res. 2017;77(4).

34. NCCN Breast Cancer (Version 5.2020). 7/21/2020. Available from: https://www.ncen.org/professionals/physician_gls/default. aspx\#breast.

35. Burstein HJ, et al. Estimating the benefits of therapy for early-stage breast cancer: the St. Gallen International Consensus Guidelines for 
the primary therapy of early breast cancer 2019. Ann Oncol. 2019;30(10):1541-57.

36. Eifel P, Axelson JA, Costa J, Crowley J, Curran WJ Jr, Deshler A, et al. National Institutes of Health Consensus Development Conference Statement: adjuvant therapy for breast cancer, November 1-3, 2000. J Natl Cancer Inst. 2001;93(13):979-89.
37. Steenbruggen TG, van Ramshorst MS, van Werkhoven E, et al. Adjuvant chemotherapy in small node-negative triple-negative breast cancer (TNBC). J Clin Oncol. 2019;37:536.

Publisher's Note Springer Nature remains neutral with regard to jurisdictional claims in published maps and institutional affiliations. 